Rev. SINAPSIS, Vol. 5, No 2, Diciembre 2014

ISSN $1390-7832$

\title{
Deficiencias del uso efectivo de las bibliotecas escolares
}

\section{Uso de la bibliotecas}

Ruth Isabel Cobos Campoverde. Mg.Sc.

Instituto Tecnológico Superior de Portoviejo, Ecuador

Contacto: ruthcampo@gmail.com

Receptado: 09/07/2014 Aceptado: 04/09/2014

\section{Resumen}

La lectura en la etapa escolar es supremamente importante y la biblioteca ha cumplido una especial labor en la enseñanza y el aprendizaje, pues esta ejerce una influencia sobre el logro académico en los estudiantes, sin embargo estas no siempre son utilizadas con la eficiencia y eficacias pertinentes, tal es el caso del uso que se le da a la biblioteca de la Unidad Educativa Informática. El objetivo de esta experiencia fue Investigar la incidencia del uso de la biblioteca en el proceso de enseñanzaaprendizaje en dicha unidad educativa. Para la puesta en marcha de este trabajo se encuestaron 112 estudiantes con el propósito de comprobar el uso eficaz de las nuevas tecnologías a través de la biblioteca escolar. Se concluyó que hay falta de gestión administrativa para dotar de insumos informáticos a la biblioteca de la institución lo que trae como resultado la poca motivación por lo que existe poca espontaneidad del estudiante por ingresar a la misma.

Palabras claves: biblioteca, influencia, enseñanza-aprendizaje- estudiantes- informático

\section{Deficiencies of the effective use of school libraries}

\begin{abstract}
The reading on the school stage is extremely important and the library has played a special role in teaching and learning, as this has an influence on academic achievement in students, but these are not always used with the efficiency, such is the case of the use that is given to the library In the Computer Educational Unit. The objective of this experiment was to investigate the impact of library use in the teaching-learning process in this Educational Unit. For the implementation of this study there was applied survy to 112 students in order to verify the effective use of new technologies through the school library. It was concluded that there is a lack of administrative management computer to provide inputs to the library of the institution, resulting in the low motivation and the little spontaneity of student library use.
\end{abstract}


Rev. SINAPSIS, Vol. 5, No 2, Diciembre 2014

ISSN $1390-7832$

Keywords: library, influence, teaching-learning-students- computers.

\section{Introducción}

El objetivo supremo de la educación es lograr que los estudiantes asuman cabalmente los retos de la época actual y participen activamente en el desarrollo económico y social de su país (Hurruitiner, 2008). Para que esto suceda, se ha tomado como tarea de primordial importancia la decisión de elevar el papel de las bibliotecas escolares como medio de trabajo sin el cual no se logra una formación integral del estudiantado.

La biblioteca escolar ofrece servicios de aprendizaje, libros y otros recursos que permiten a todos los miembros de la comunidad escolar forjarse un pensamiento crítico y utilizar eficazmente la información en cualquier formato y medio de comunicación. Las bibliotecas escolares están conectadas con la vasta red de bibliotecas e información, de acuerdo con los principios del Manifiesto de la UNESCO sobre la Biblioteca Escolar.

Se precisan cambios de las bibliotecas escolares en su dimensión física, así como

enfocar su organización no en base a una función tradicional de depósito sino más bien

en la articulación y generación de servicios mediadores. Los servicios nuevos más

relevantes resultan: las acciones de filtro y redistribución de contenidos, la generación

de entornos informacionales de aprendizaje y la administración de plataformas

virtuales de interacción social... (Duran, 2013)

Frente a la situación observada en la Unidad Educativa Particular Informática "Portoviejo" respecto al deficiente uso de la biblioteca de la institución por parte del estudiante y de los docentes, surgió la necesidad de llevar a cabo una investigación, la misma que estuvo encaminada a conocer la repercusión del deficiente uso adecuado a la biblioteca y su problemática.

El presente trabajo recoge los resultados de dicha investigación, buscando explicar cuáles son las causas que impiden visitar el departamento bibliotecario con los fines pertinentes por parte de los estudiantes, que nivel de interés manifiestan los docentes de la institución con respecto a la biblioteca y trabajos investigativos para motivar a los estudiantes al uso adecuado de esta para un aprendizaje más efectivo. 
Rev. SINAPSIS, Vol. 5, No 2, Diciembre 2014

ISSN $1390-7832$

La investigación se fundamentó en las teorías filosóficas de Jean Piaget cuyos principios orientan las conclusiones obtenidas en las mismas.

El trabajo visualiza como la biblioteca requiere de un alma y de un espacio. La biblioteca debe respirar libros y lectores. Refleja el pulso de un centro, el grado de compromiso personal con la lectura, la unión de los maestros y profesores de todas las áreas, la búsqueda de nuevos métodos de enseñanza, en definitiva, representa un sueño, donde es de vital importancia vital el equipamiento moderno para el desarrollo de esta en el mundo actual.

\section{Materiales y métodos}

\section{Lugar y períodos de desarrollo del experimento.}

La investigación se realizó en la Unidad Educativa Informática en la ciudad de Portoviejo en la provincia de Manabí, Ecuador. Se tomaron los resultados de las encuestas realizadas a estudiantes y profesores en el curso académico 2011-2012.

\section{Muestra}

Para la puesta en marcha de la investigación, se utilizaron 112 de estudiantes y 54 profesores de la Unidad Educativa Informática. Esta muestra fue considerada de tipo probabilístico; ya que se necesitó de la aplicación de la Estadística Inferencial por el manejo que se le dio a la información.

\section{Aspectos teóricos}

Frente a los tradicionales libros, vídeos y juegos, los nuevos contenidos educativos creados con recursos tecnológicos permiten presentar la información de otra forma. Los contenidos se hacen más dinámicos (interactividad) más atractivos (presentación simultánea de texto, sonidos e imágenes) y más variados. Estas nuevas prestaciones pueden facilitar el aprendizaje y permitir mejoras cognitivas sobre todo de los alumnos con dificultades, al aplicar metodologías más activas y menos expositivas.

Las TIC han permitido que los contenidos educativos no sólo los creen los productores sino que también sean los propios profesores o incluso los alumnos quienes, solos o trabajando en grupo, desarrollen contenidos curriculares propios o adapten los de otros. En este sentido, 
Rev. SINAPSIS, Vol. 5, No 2, Diciembre 2014

ISSN $1390-7832$

cabe decir que los materiales didácticos se han multiplicado, sobre todo teniendo en cuenta que la tendencia es la de la convivencia de los materiales tradicionales con los nuevos.

Sin embargo, la creación de contenidos no es tan sencilla como transponer un libro a una pantalla, sino que la dificultad radica en ofrecer algún valor añadido, como la posibilidad de interactuar o presentar simulaciones o realidad virtual o incluso adaptaciones de los materiales a las características nacionales, regionales e incluso lo-cales. Por otra parte, los nuevos contenidos resultan más adaptables y se modifican con mayor facilidad.

Los profesores tienen la oportunidad de generar contenidos educativos de acuerdo con los intereses o las particularidades de sus alumnos y de su contexto educativo. Es-tos contenidos, debido a su escaso coste, pueden crearse para grupos de alumnos reducidos o incluso para algún alumno en particular.

Esta facilidad en la creación de materiales educativos ha dado lugar, sobre todo en algunos países, a la existencia de un mayor volumen de contenidos, lo que ha planteado el problema de la evaluación de los mismos.

Así, países como EE.UU., Suecia, Francia o Reino Unido han instaurado sistemas de evaluación de los materiales didácticos, casi siempre desarrollados a través de grupos de expertos. Esta preocupación nos lleva de nuevo a re-afirmar el papel crítico del docente que debería ser capaz de evaluar si los contenidos son aptos o no para la enseñanza.

Así debe ser en los países de Latinoamérica.

Según Piedrahita (2009) los gobiernos de América Latina tienen ahora la gran oportunidad en la trasformación de sus sistemas educativos; de mejorar la calidad de sus escuelas; de reducir la inequidad en las oportunidades que se ofrecen a los jóvenes en los diferentes medios bibliotecarios...

La investigación arrojó que para que se haga efectivo el uso de las bibliotecas escolares en Ecuador se debe cumplir varios aspectos al respecto registrados en la Constitución de la Republica.

Corresponde a las Administraciones educativas dotar a los centros públicos de los medios materiales y humanos necesarios para ofrecer una educación de calidad y garantizar la igualdad de oportunidades en la educación. 
Rev. SINAPSIS, Vol. 5, No 2, Diciembre 2014

ISSN $1390-7832$

En el contexto de lo dispuesto en el apartado anterior, los centros dispondrán de la infraestructura informática necesaria para garantizar la incorporación de las tecnologías de la información y la comunicación en los procesos educativos. Corresponde a las Administraciones educativas proporcionar servicios educativos externos y facilitar la relación de los centros públicos con su entorno y la utilización por parte del centro de los recursos próximos, tanto propios como de otras Administraciones públicas.

Los centros que escolaricen alumnado con necesidad específica de apoyo educativo, en proporción mayor a la establecida con carácter general o para la zona en la que se ubiquen, recibirán los recursos complementarios necesarios para atender adecuadamente a este alumnado.

Las Administraciones educativas facilitarán que aquellos centros que, por su número de unidades, no puedan disponer de los especialistas a los que se refiere el artículo 93 de esta Ley, reciban los apoyos necesarios para asegurar la calidad de las correspondientes enseñanzas.

Las Administraciones educativas potenciarán que los centros públicos puedan ofrecer actividades y servicios complementarios a fin de favorecer que amplíen su oferta educativa para atender las nuevas demandas sociales, así como que puedan disponer de los medios adecuados, particularmente de aquellos centros que atiendan a una elevada población de alumnos con necesidad específica de apoyo educativo.

Las bibliotecas escolares tienen una gran influencia en los resultados del aprendizaje, pues el desarrollo de las habilidades lectoras complementadas con el uso de los recursos pueden dar mejores resultados a la hora de investigar un tema o realizar una exposición, puesto que son complementarias tanto a las explicaciones del profesor como de búsqueda de temas de interés o de temas no comprendidos.

Alcántara (2008) "Es indudable el apoyo que el Estado debe prestar para crear una conducta lectora, no se debe pasar por alto la importancia que tienen las escuelas y las bibliotecas para con ésta por lo cual la colaboración del Estado para el sistema de educación es un hecho... que debe ser reconocido, pero ese apoyo debe llevar a la creación y subsidio económico a todo tipo de bibliotecas..." (s.n) 
- Los centros de enseñanza dispondrán de una biblioteca escolar.

- Las Administraciones educativas completarán la dotación de las bibliotecas de los centros públicos de forma progresiva. A tal fin elaborarán un plan que permita alcanzar dicho objetivo dentro del periodo de implantación de la presente Ley.

- Las bibliotecas escolares contribuirán a fomentar la lectura y a que el alumno acceda a la información y otros recursos para el aprendizaje de las demás áreas y materias y pueda formarse en el uso crítico de los mismos. Igualmente, contribuirán a hacer efectivo lo dispuesto en los artículos 19.3 y 26.2 de la presente Ley.

- La organización de las bibliotecas escolares deberá permitir que funcionen como un espacio abierto a la comunidad educativa del centro respectivo.

- Los centros podrán llegar a acuerdos con los municipios respectivos, para el uso de bibliotecas municipales con las finalidades previstas en este artículo.

\section{Resultados del análisis estadístico}

Para obtener la información una vez que el Rector de la Institución autorizó la realización de las encuestas se procedió a aplicarlas. Se trabajó con sustento en los análisis de los datos obtenidos de los instrumentos de investigación durante el proceso de recolección de la información.

Las encuestas se las realizó de forma anónima a docentes y estudiantes, para determinar con mayor precisión datos con certeza y autenticidad con el afán de obtener resultados reales.

Una vez realizadas las encuesta y recopilada la información necesaria se procedió a la tabulación e interpretación de los resultados, para luego efectuar una propuesta que dé solución al problema planteado.

TABLA No 1

ORDEN

ALTERNATIVAS

$\mathbf{F}$

$\%$ 

a.
$\mathrm{Si}$
68
$61,00 \%$

\begin{tabular}{cccc}
\hline b. & No & 44 & $39,00 \%$ \\
\hline TOTAL & 112 & $100 \%$ \\
\hline
\end{tabular}

\section{GRÁFICO No 1 \\ La biblioteca cuenta con un ambiente y tecnología para la investigación}

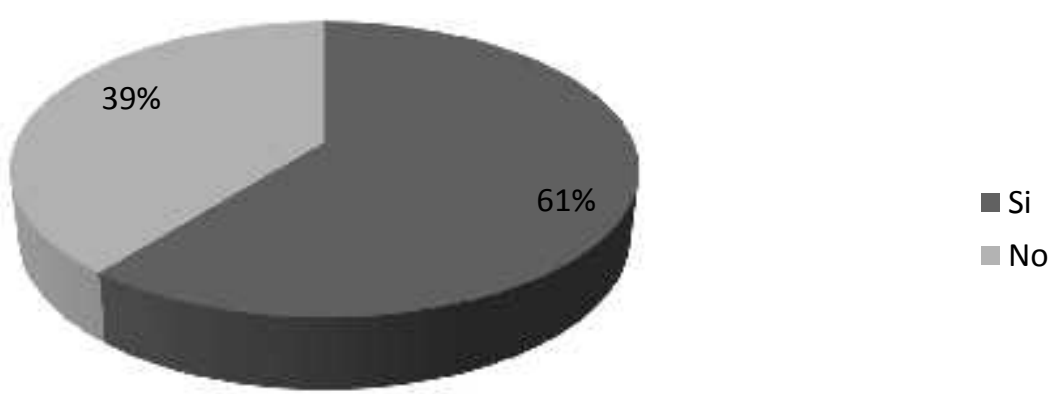

De acuerdo a la encuesta aplicada a 112 estudiantes, la pregunta se elaboró con el objetivo de investigar con qué ambiente y tecnología cuenta la biblioteca en el que se puedan realizar todas las actividades de investigación, se obtuvo el siguiente resultado de 68 estudiantes que representan el $61 \%$ señalan que sí y 44 estudiante que corresponden al 39\% que no está de acuerdo con lo establecido.

Referente a estos resultados dejan ver con claridad que en su mayoría los estudiantes consideran que si cuenta la biblioteca con un ambiente y tecnología adecuada.

Piaget (1980) Abogó explícitamente por una pedagogía experimental Protestó enérgicamente que en un terreno tan accesible a la experimentación... el pedagogo no organice experiencias continuadas y metódicas y se contente con resolver las cuestiones a base de opiniones que "el buen sentido" recubre, de hecho, más de razones afectivas que efectivas.

Los resultados a esta pregunta demuestran claramente que la motivación es un requisito importante y una valiosa herramienta que el docente debe aplicar para generar el interés en los estudiantes para 
Rev. SINAPSIS, Vol. 5, No 2, Diciembre 2014

aprender. Hoy en día existen muchos instrumentos tecnológicos que pueden ser utilizados, además de los tradicionales como la lectura para así lograr el éxito en todos los emprendimientos.

\section{TABLA $\mathbf{N}^{\circ} 2$}

\begin{tabular}{lll}
\hline ORDEN & ALTERNATIVAS & F
\end{tabular}

$\begin{array}{lll}\text { a. } & \text { Siempre } & 13\end{array}$
b.
A veces
29
$54,00 \%$

c.

Rara vez

9

$17,00 \%$

d.

Nunca

3

$5,00 \%$

TOTAL

54

$100 \%$

Fuente: Docentes de la Unidad Educativa Informática "Portoviejo"

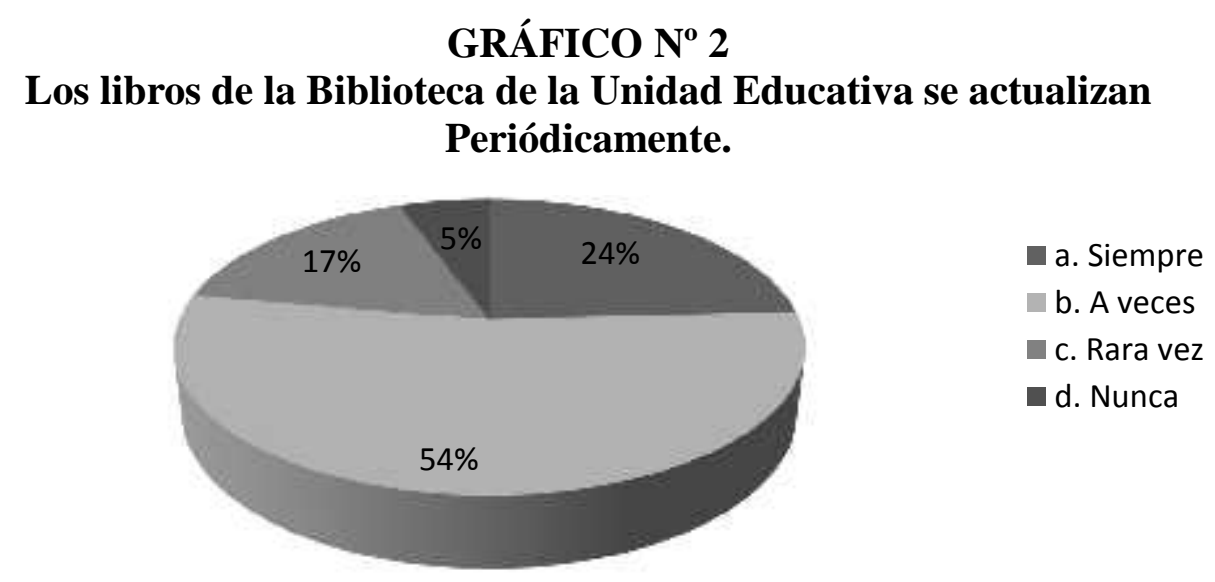

La finalidad de esta pregunta es identificar la realidad del departamento bibliotecario con respecto a la actualización de los textos, se pudo determinar que 13 de los encuestados que 
Rev. SINAPSIS, Vol. 5, No 2, Diciembre 2014

ISSN $1390-7832$

corresponden al 24\% manifestó que siempre se actualiza; 29 docentes con el 54\% respondieron que a veces; nueve de los encuestado que corresponde al $17 \%$ rara vez y tres que representa el $5 \%$ nunca.

Ante lo expuesto de ciertos docentes nos hace pensar que ellos jamás visitan la biblioteca para realizar investigaciones o revisar materiales de trabajo y hacen un comentario fuera de la realidad.

Piaget exploró las aplicaciones de su teoría en los aspectos de cognición, inteligencia y desarrollo moral. Muchas de sus investigaciones se basaron en el desarrollo de los conceptos lógicos y matemáticos, Los materiales y las actividades de aprendizaje deben estar apropiados para la edad del niño, tomando en cuenta su capacidad de operaciones mentales o motrices, evitando así pedirles a los alumnos que lleven a cabo tareas que van más allá de su desarrollo cognitivo. Utilizar métodos de enseñanza que involucren activamente a los estudiantes y les presenten retos (Piaget, 1896)

Los docentes deben de interesarse en los textos y el material existente de la biblioteca para poder utilizarlos en los estudiantes.

\section{Discusión}

Los resultados arrojados globalmente en la investigación, coinciden con los realizados por otros investigadores que refieren sobre la importancia de las nuevas tecnologías en las bibliotecas escolares, los cuales plantean que el desarrollo de la tecnología en los últimos tiempos y la generalización, uso y aplicación están provocando una revolución con profundos cambios en la sociedad (María, 2012; Todd, 2012; Pérez, 206)

De esa manera se corrobora a través de diferentes fuentes sobre la necesidad que tanto los diferentes aparatos gubernamentales, así como las direcciones de las instituciones deben prestar especial atención al equipamiento con los modernos equipos tecnológicos que. "Yo veo la Biblioteca Escolar de Calidad (BEC) como el sitio de encuentro físico y virtual de la Información que se convierte en conocimiento, donde el alfabetismo, la indagación, la reflexión, la imaginación, el descubrimiento y la creatividad son fundamentales... (Todd, 2012, s.n)

\section{Conclusiones}


Rev. SINAPSIS, Vol. 5, No 2, Diciembre 2014

Se concluyó que la gestión administrativa de parte de las autoridades es muy pobre, en cuanto

a la implementación de un control del trabajo en la biblioteca, lo que conlleva a que el nivel cultural de los estudiantes es intermedio en el aspecto de la investigación. Una gran cantidad de proyectos educativos se pueden implementar en el aula de clase, para así fomentar las visitas a las bibliotecas, mostrándole al estudiante las ventajas que se obtienen al hacer de esto un hábito, proyectos que pueden facilitar el mejoramiento e interés de hacer más dinámico todo trabajo de investigación, donde la nuevas tecnologías de la información son de vital importancia.

\section{Bibliografía}

1. Alcántara, M. (2008). Las bibliotecas escolares, importante recurso. Innovación y Experiencia Educativas, (13).

2. Hurruitiner, S. (2008) La primera idea rectora: la unidad entre la educación y la Instrucción. Capitulo II, acápite 2.2.1. En: La universidad cubana: el modelo de formación. Editorial Félix Varela. 1ra ed., 26. La Habana. ISBN 9592588945

3. Pérez, E. (2006). Biblioteca escolar digital. Recuperado en 26 de noviembre de 2014 en http://www.anabad.org/archivo/docdow.php?id=158

4. Piedrahita, F. (2009). El porqué de las TIC en la educación. Edukateka, 2, (11), 25 -27.

5. Rodríguez, M. (2012). Educación Biblioteca y TIC en la sociedad de la información: reto y compromiso. Recuperado el 26 de noviembre de 2014 el 26 de noviembre de 2014 en http://www.web.upsa.es/spdece08/contribuciones/151_poster_BibliotecaEscolarDig ital.pdf

6. Sarabia, T. (2012). Estrategias metodológicas para el perfeccionamiento del proceso de enseñanza aprendizaje de matemática, en los séptimos años de educación general básica de la Escuela Nocturna "Vicente Anda Aguirre".

7. Torres, W. (2009) "La mediación pedagógica y su influencia en el proceso enseñanza aprendizaje de los estudiantes de la carrera de parvulario modalidad semipresencial de la universidad técnica de Ambato periodo 2007. 